Cahiers $d u$ MONDE RUSSE

\section{Cahiers du monde russe}

Russie - Empire russe - Union soviétique et États indépendants

45/3-4 | 2004

Varia

\title{
Tat'jana Smirnova, « Byvšie ljudi » Sovetskoj Rossii
}

\section{Nathalie Moine}

\section{OpenEdition \\ Journals}

Édition électronique

URL : https://journals.openedition.org/monderusse/4199

DOI : 10.4000/monderusse.4199

ISSN : $1777-5388$

\section{Éditeur}

Éditions de l'EHESS

\section{Édition imprimée}

Date de publication : 1 juillet 2004

Pagination : 692-695

ISBN : 2-7132-2009-2

ISSN : $1252-6576$

\section{Référence électronique}

Nathalie Moine, «Tat'jana Smirnova, « Byvšie ljudi » Sovetskoj Rossii », Cahiers du monde russe [En ligne], 45/3-4 | 2004, mis en ligne le 03 juin 2009, consulté le 03 septembre 2022. URL : http:// journals.openedition.org/monderusse/4199; DOI : https://doi.org/10.4000/monderusse.4199

Ce document a été généré automatiquement le 3 septembre 2022

Tous droits réservés 


\title{
Tat'jana Smirnova, « Byvšie ljudi » Sovetskoj Rossii
}

\author{
Nathalie Moine
}

\section{RÉFÉRENCE}

Tat'jana SMIRNOVA, « Byvšie ljudi » Sovetskoj Rossii. Strategii vyživanija i puti integracii, 1917-1936 gody (Les « ci-devant » de la Russie soviétique. Stratégies de survie et voies d'intégration, 1917-1936). Moscou, « Mir istorii », 2003, 296 p.

1 S'inscrivant dans la veine d'une histoire sociale en plein renouveau chez les jeunes historiens russes, Tat'jana Smirnova se propose de revenir sur le sort des «ci-devant " (byvšie) de la révolution bolchevique. Alors que cette expression est omniprésente dans le vocabulaire des années post-révolutionnaires, les anciens "privilégiés " du régime tsariste sont des oubliés de l'historiographie, tant soviétique qu'occidentale. Les travaux portant sur le démantèlement $d u$ système social et économique dont héritent les bolcheviks s'attachaient uniquement à décrire les décisions politiques et traitaient de façon abstraite de leurs victimes, le thème de la lutte contre les "classes exploiteuses » et de la construction d'une « société sans classes (antagonistes) » ne laissant aucune place à l'expérience humaine.

2 Tat'jana Smirnova entend donc revenir sur cet oubli et propose un programme de recherche passionnant: quelle fut l'expérience concrète des byvšie dans la tourmente révolutionnaire, puis dans les premières années de la construction du socialisme, l'ouvrage s'arrêtant à la date de l'adoption d'une nouvelle Constitution pour l'Union Soviétique -1936? Quel regard portaient ces «ci-devant» sur le nouveau régime, jusqu'à quel degré et par quels canaux s'intégrèrent-ils dans la nouvelle société?

3 Dès le départ, l'auteur souligne l'ambiguïté du propos : qui sont, après tout, ceux que l'on appelle les byvšie ? En effet, bien au-delà des anciennes élites tsaristes, ce terme désigne tout autant des représentants des anciennes classes moyennes, voire, par extension, des 
personnes aux origines prolétariennes irréprochables, mais dont les activités présentes sont fustigées comme des résidus inacceptables de l'Ancien Régime.

Cette ambivalence est en réalité au cœur du travail de Tat'jana Smirnova, qui relève l'usage très politique du terme dans les premières années de la Révolution: les membres du Parti S.-R. sont ainsi nommés, indépendamment de leurs origines sociales, par A. V. Lunačarskij dans un ouvrage de 1922 intitulé Byvšie ljudi : očerk istorii partii es-erov (Les «ci-devant» : essai d'histoire du parti des S.-R.), alors même que de nombreux leaders bolcheviks n'ont de prolétarien que l'idéologie.

5 Cependant, l'ouvrage ne s'attarde guère sur cette question pour se concentrer sur l'infinie déclinaison de l'appartenance de classe dans le contexte soviétique de l'entre-deuxguerres. Est-elle affaire de parenté, de niveau d'éducation, de genre de vie, de patrimoine? Smirnova se plaît à montrer, notamment à travers une grande richesse lexicale, l'extraordinaire confusion des esprits sur ce problème pourtant central puisque la nécessité d'une « répression sans pitié des exploiteurs » est inscrite au cœur des textes fondamentaux du nouvel État.

6 L'ensemble de ces remarques fait écho aux travaux récents sur les lišency (privés de droits civiques), dont une partie, mais une partie seulement, est définie, dans les textes mettant en place cette mesure discriminatoire, comme d'anciens maillons du régime tsariste et écartés comme tels du corps civique ${ }^{1}$.

7 Cependant, l'étude s'étend sur de nombreux autres aspects de la discrimination envers les byvšie. On notera tout particulièrement les développements concernant la politique du logement, c'est-à-dire la «municipalisation » d'un certain nombre d'immeubles à partir de 1918, suivie de leur démunicipalisation partielle en 1920. À partir de cas individuels trouvés dans les archives, Smirnova montre ainsi combien la « bacchanale des expulsions et des municipalisations » se développe de façon anarchique sans vraiment tenir compte des instructions de départ définissant ceux qui devaient être soumis à cette mesure. Le même type d'archives concernant la démunicipalisation reflète à nouveau l'arbitraire des pouvoirs locaux, et leur vénalité, dans un contexte où les budgets locaux sont totalement dépendants de la ponction exercée sur les byvšie, mais montre aussi la persistance de certains liens entre ces derniers, devenus locataires, et leurs biens. Liens fragiles, à la merci d'une décision d'expulsion, mais qui témoignent tout de même de la conservation minimale de repères, dans un océan de migrations et de morts.

8 De fait, la question des hautes élites du régime est assez rapidement évacuée, puisque celles-ci ont très vite quitté le pays. Selon Smirnova les victimes, tant de la Terreur Rouge lors de la guerre civile que des mesures discriminatoires moins violentes, sont le menu fretin - anciens membres de la petite et moyenne bourgeoisie tsariste.

9 La Terreur Rouge, officiellement lancée à l'automne 1918, s'accompagne d'une violente campagne de haine et d'enrôlement des byvšie dans le travail. Elle marque aussi les premières tentatives d'enregistrement de catégories de la population selon les critères du nouveau régime, telle que celle des "personnes ayant anciennement appartenu à l'état de bourgeois et de fonctionnaires de l'État (« registracija lic byvšego buržuaznogo $i$ činovnogo sostojanjia »). Outre les problèmes de définition, se pose aussi la question de la gestion des informations collectées sur les individus.

10 En réalité, l'intérêt principal des passages sur les années 1920 tient dans les éléments qui préfigurent les grandes campagnes répressives staliniennes. Ainsi de la discussion autour des papiers d'identité ou des projets rédigés au tournant des années 1923-1924 concernant 
une purge massive de Moscou de ces éléments socialement dangereux, dont on établit la nomenclature. Si le projet est finalement jugé nul et non avenu, les autorités moscovites, frustrées d'une opération de grande ampleur, n'en recourent pas moins périodiquement à des déportations individuelles, sur décision de la GPU ou du département administratif du Mossovet. L'introduction d'un passeport intérieur unique pour la population urbaine soviétique à la fin de 1932, puis les expulsions massives qui s'ensuivent, sont peu étudiées dans le livre mais constituent bien une conséquence directe des atermoiements de la NEP. L'opération «Byvšie ljudi» qui frappe la population de Leningrad en 1935 est également évoquée.

11 La dernière partie, consacrée au sort des enfants de byvšie, brosse un tableau extrêmement nuancé du comportement des pouvoirs publics, mais aussi de leur véritable capacité à connaître le passé de leurs administrés. Smirnova établit une distinction entre les jeunes qui arrivent adolescents ou à peine adultes à la Révolution et sont donc frappés de la même discrimination que les adultes, et les enfants qui, au contraire, ne font pas l'objet d'un tel acharnement, au moins jusqu'au milieu des années 1920. L'auteur soutient que le régime, dans ses premières années, tient au principe de totale égalité entre les enfants, jugés irresponsables de leurs origines sociales. Plus tard, les différences de traitement en matière d'allocation de la nourriture et d'autres biens se feront selon des critères de proximité avec le pouvoir, et non d'origine sociale. Différents modes d'intégration à la société soviétique sont ensuite envisageables, sans être toujours totalement fiables. Parmi eux, le passage par l'Armée Rouge ou l'usine, qui permet par la suite de fréquenter un établissement d'enseignement supérieur. Si les enseignants du début des années 1920 sont accablés par le très faible niveau de cohortes d'étudiants massivement prolétaires, il n'en reste pas moins que certaines facultés, notamment les départements artistiques et pédagogiques, sont majoritairement remplies d'enfants héritiers d'une tradition familiale. La haine de classe qui se déploie à la fin des années 1920 vient fortement perturber ces stratégies d'intégration - ou de maintien - dans la société. Les manifestations du pouvoir en faveur de l'émancipation des jeunes vis-à-vis de leurs parents contrastent avec les purges massives qui touchent indistinctement toutes les générations.

La conclusion de cette partie préfigure celle de l'ensemble de l'ouvrage : la situation des byvšie et de leurs enfants est difficile, en proie aux contradictions du régime, mais elle n'est pas toujours désespérée. Contrairement à une historiographie actuelle qui aurait tendance à considérer l'origine sociale comme une tâche indélébile dans le contexte soviétique de l'entre-deux-guerres et à attacher trop d'importance aux procédures d'enquête administrative sur le passé des citoyens soviétiques, Smirnova insiste sur l'extrême fluidité des situations : du fait du désordre de la bureaucratie soviétique, les possibilités de passer à travers les mailles du filet sont légion. Lorsque le pouvoir se durcit à nouveau et que commencent les purges de la Grande Terreur, la population, passée au creuset de la révolution, de la guerre civile et de l'industrialisation, ne contient plus de groupe social directement hérité de la Russie tsariste. À la fin des années 1930, l'origine sociale est désormais le prétexte, et non plus la cause principale, de la répression, et ce sont bien les ouvriers et les paysans qui se retrouvent en première ligne de la terreur stalinienne.

14 L'ouvrage de Smirnova touche ainsi à des problématiques aujourd'hui centrales dans la compréhension des premières décennies du régime. Les aspects les mieux traités sont ceux qui ont bénéficié de sa fréquentation des archives, notamment de la ville de Moscou et de sa région, mais aussi des archives centrales pour la première décennie suivant la 
révolution, alors que d'autres sont traités de façon plus superficielle et servent plutôt de pistes de recherche fructueuses, en particulier ce qui concerne le sort des enfants.

Certaines questions méthodologiques restent à résoudre : ainsi, un cas individuel retrouvé dans les archives constitue une source inépuisable d'informations sur les acteurs sociaux qui manquent tant dans l'historiographie traditionnelle sur l'Union soviétique, mais il ne dit rien sur un groupe dans son ensemble. De fait, le flou dans lequel les autorités tout comme l'auteur laissent la notion de byvšie, interdit de tirer une véritable conclusion sur l'ampleur de la répression qui aurait pu les frapper. Il n'en est pas moins important de souligner, à l'instar de Smirnova, que la préoccupation essentielle du pouvoir concerne bien les " éléments socialement dangereux", définis avant tout par leur situation présente, catégorie qui rassemble les membres d'un monde semi-criminel et les personnes non engagées dans un travail socialement productif - les byvšie se trouvant ainsi à l'intersection de ce vaste contingent de personnes suspectes. On peut alors remettre en cause la pertinence de 1936 comme année de conclusion de cette étude. L'auteur cite elle-même les données du goulag de 1942 mentionnant les "ex-propriétaires fonciers, fabricants, commerçants» comme preuve d'une importance résiduelle apportée à la lutte des classes, sans préciser qu'il s'agit à coup sûr de nouveaux byvšie, en provenance des régions occidentales annexées en 1939-1940.

\section{NOTES}

1. V. I. Tihonov, V.S. Tiažel'nikova, I. F. Jušin, Lišenie izbiratel'nyh prav v Moskve v 1920-1930-e gody. Novye arhivnye materialy $i$ metody obrabotki, Moscou, Izdatel'stvo Ob"edinenija Mosgorarkhiv, 1998; Golfo Alexopoulos, Stalin's outcasts: aliens, citizens, and the Soviet state, 1926-1936, Ithaca, Cornell University Press, 2003. 